\title{
Bromobenzoylation of Methyl $\alpha$-D-Mannopyranoside: Synthesis and Spectral Characterization
}

\author{
Farhana Yasmin a, Md R. Amina, \\ Anowar Hosen' and Sarkar M.A. Kawsar*a \\ ${ }^{a}$ Laboratory of Carbohydrate \\ and Nucleoside Chemistry (LCNC) \\ University of Chittagong \\ Chittagong, Bangladesh \\ ${ }^{b}$ Centre for Advanced Research in Sciences \\ University of Dhaka \\ Dhaka, Bangladesh
}

Received 11.04.2021, received in revised form 12.05.2021, accepted 05.06.2021

\begin{abstract}
The widening importance of carbohydrate derivatives as unrivaled potential antimicrobial and therapeutic drugs has attracted attentionto the synthesis of mannopyranoside derivatives. In the present study, regioselective 3-bromobenzoylation of methyl $\alpha$-D-mannopyranoside (1) was carried out using the direct method and gave the corresponding 6-O-(3-bromobenzoyl) derivative (2) in excellent yield. A number of 2,3,4-tri- $O$-acyl derivatives (3-10) of this 6 -substitution product using a wide variety of acylating agents were also prepared in order to obtain newer derivatives of synthetic and biological importance. The chemical structures of the newly synthesized compounds were ascertained by analyzing their physicochemical, elemental, and spectroscopic data. Additionally, the X-ray powder diffraction (XRD) of these acylated products was studiedfor quantitatively identifying crystalline compounds.Therefore, due to the importance of carbohydrates, it might be useful to develop a good method for the synthesis of carbohydrate-based drugs of the current global situation for health and disease.
\end{abstract}

Keywords: methyl $\alpha$-D-mannopyranoside, benzoylation, derivatives, spectroscopy, XRD.

Citation: Yasmin F., Amin Md R., Hosen A., Kawsar S.M.A. Bromobenzoylation of methyl $\alpha$-d-mannopyranoside: synthesis and spectral characterization, J. Sib. Fed. Univ. Chem., 2021, 14(2), 171-183. DOI: 10.17516/1998-2836-0226

(C) Siberian Federal University. All rights reserved

This work is licensed under a Creative Commons Attribution-NonCommercial 4.0 International License (CC BY-NC 4.0).

* Corresponding author E-mail address: akawsarabe@yahoo.com 


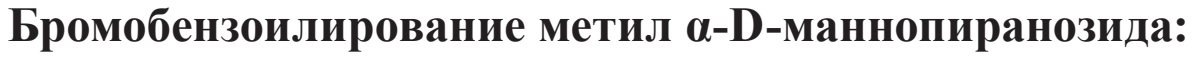 синтез и спектральная характеристика
}

\author{
Ф. Ясмин ${ }^{\mathrm{a}}$ Мд.Р. Амин ${ }^{\mathrm{a}}$, \\ А. Хосен ${ }^{\sigma}$, С.М.А. Кавсар ${ }^{\mathrm{a}}$ \\ а Лаборатория химии углеводов и нуклеозидов (LCNC) \\ Университет Читтагонга \\ Бангладеш, Читтагонг \\ ${ }^{6}$ Центр передовых научных исследований \\ Даккский университет \\ Бангладеш, Дакка
}

\begin{abstract}
Аннотация. Растущее значение производных углеводов как ценных потенциальных антимикробных и терапевтических препаратов привлекло внимание к синтезу производных маннопиранозида. В настоящем исследовании было выполнено региоселективное 3-бромбензоилирование метил- $\alpha-\mathrm{D}$-маннопиранозид (1) прямым методом и получено соответствующее производное 6-O-(3-бромбензоила) (2) с высоким выходом. Ряд 2,3,4-три- $O$-ацильных производных (3-10) указанного 6-замещенного продукта с использованием широкого спектра ацилирующих агентов был также создан с целью получения новых производных, имеющих синтетическую и биологическую важность. Химические структуры новых синтезированных соединений установлены путем анализа их физико-химических, элементных и спектроскопических данных. Кроме того, для количественной идентификации кристаллических соединений исследована рентгеновская порошковая дифракция (XRD) указанных ацилированных продуктов. Таким образом, учитывая важность углеводов, было бы полезно разработать надежный метод синтеза препаратов на основе углеводов в современной глобальной ситуации относительно вопросов здоровья и заболеваний.
\end{abstract}

Ключевые слова: метил- $\alpha-\mathrm{D}$-маннопиранозид, бензоилирование, производные, спектроскопия, рентгеновская дифракция.

Цитирование: Ясмин, Ф. Бромобензоилирование метил $\alpha$-D-маннопиранозида: синтез и спектральная характеристика / Ф. Ясмин, Мд. Р. Амин, А. Хосен, С.М.А. Кавсар // Журн. Сиб. федер. ун-та. Химия, 2021, 14(2). С. $171-183$. DOI: $10.17516 / 1998-2836-0226$

\section{Introduction}

Carbohydrates are an important class of natural products that belongs to the class of organic compounds found in living organisms on earth. They are involved in several processes of life such as glycolysis or glucogenesis [1]. In addition to their role as an energy source, they also play an important role in several biological signaling and recognition processes, such as immune response, inflammatory reactions, cancer metathesis and viral infections [2, 3]. Carbohydrates are frequentbuilding blocks for the synthesis of drugs. Since carbohydrates are highly functionalized molecules with several 
stereocenters, their de novo synthesis is a challenging issue although, an arsenal of methods have been developed to facilitate their production. Unfortunately, most of the approaches are lengthy and require sophisticated protecting-group strategies [4].

All these processes are potential targets for therapeutic intervention and carbohydrate-based drugs are rapidly being engaged by the modern biotechnology and pharmaceutical industry [5]. Chemists and biochemists have developed new methods to rapidly synthesize oligosaccharides, enabling them to generate complex polysaccharides and analogs of natural products. However, carbohydrate researchers consider selective acylation as one of the most important and versatile methods for the protection of the hydroxyl groups. Various methods for selective acylation have so far been developed and successfully employed in carbohydrate chemistry [6-8]. Of these, the direct method is considered as one of the most effective and versatile [9].

From the literature survey, it was revealed that a large number of biologically active compounds contain aromatic, heteroaromatic and acyl substituents $[10,11]$. Nitrogen, sulphur, and halogencontaining substituents are also known to enhance the biological activity of the parent compound $[11,12]$. It is also known that if an active nucleus is linked to another active nucleus, the resulting molecule may show greater potential for biological activity [12]. The benzene and substituted benzene nuclei play an important role as a common denominator of various biological activities [13]. From our previous works we also observed that in many cases the combination of two or more acyl substituents in a single molecular framework enhances the biological activity by manyfold than their parent nuclei [14-16].

Encouraged by our findings [17-20] and also above literature reports, we synthesized a series of methyl $\alpha$-D-mannopyranoside (1) (Fig. 1) derivatives deliberately incorporating a wide variety of probable biologically active components to the D-glucose moiety. The synthetic part is reported herefor the first time.

\section{Experimental}

\section{Materials and methods}

Melting points were determined on an electrothermal melting point apparatus and are uncorrected. Evaporation was performed under reduced pressure on a Buchi rotary evaporator. Thin-layer chromatography was performed on Kieselgel $\mathrm{GF}_{254}$ and visualization was accomplished by spraying the plates with $1 \% \mathrm{H}_{2} \mathrm{SO}_{4}$, followed by heating at $150-200{ }^{\circ} \mathrm{C}$ until colouration took place. Column chromatography was performed with silica gel $\mathrm{G}_{60} .{ }^{1} \mathrm{H}-\mathrm{NMR}(400 \mathrm{MHz})$ (unless otherwise specified)

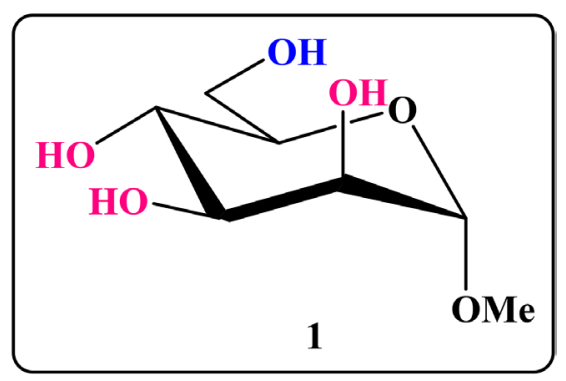

Fig. 1. Structure of themethyl $\alpha$-D-mannopyranoside (1) 
spectra were recorded for solutions in deuterochloroform (internal tetramethylsilane) with a Bruker nuclear magnetic resonance spectrophotometer. Infrared spectral analyses were recorded using a Fourier-transform infrared (FTIR) spectrophotometer (IR Prestige-21, Shimadzu, Japan) within 200$4000 \mathrm{~cm}^{-1}$. Mass spectra of the synthesized compounds were obtained by liquid chromatographyelectrospray ionization tandem mass spectrometry in positive ionization mode. All reagents used were commercially available Sigma-Aldrich (Germany) and were used as received unless otherwise specified.

\section{Synthesis}

Toward the goal of developing broadly useful strategies for organic synthesis, our research labof carbohydrate and nucleosidechemistry (LCNC) is intended to prepare a series of D-mannopyranoside derivatives for use as test compounds for biological evaluation. Additionally, over the past several years, LCNC has been actively engaged in the synthesis of carbohydrate derivatives containing various acyl groups to investigate their antibacterial, antifungal, anticancer properties with computational studies [21-23].

A solution of the methyl $\alpha$-D-mannopyranoside (1) (100 $\mathrm{mg}, 0.51 \mathrm{mmol})$ in dry $N$, $N$-dimethylaniline $(3 \mathrm{ml})$ was cooled to $-5{ }^{\circ} \mathrm{C}$ when 3 -bromobenzoyl chloride $(0.07 \mathrm{ml}, 1.1$ molar eq.) was added. The solution was stirred at $0{ }^{\circ} \mathrm{C}$ for six hours and then kept standing overnight at room temperature. The reaction mixture was shaken with a few pieces of ice and then extracted with chloroform $(3 \times 3 \mathrm{ml})$. The organic layer was washed successively with $5 \%$ hydrochloric acid, saturated aqueous sodium hydrogen carbonate solution and distilled water. The organic layer was dried $\left(\mathrm{MgSO}_{4}\right)$, filtered and the filtrate evaporated off under reduced pressure to leave a syrup. Purification of the resulting syrupy residue was achieved by silica gel column chromatography with $\mathrm{CH}_{3} \mathrm{OH} / \mathrm{CHCl}_{3}=1 / 8\left(\mathrm{v} / \mathrm{v}, R_{f}=0.51\right)$ as eluent to afford the 3-bromobenzoate derivative $2(182.6 \mathrm{mg})$ which was used in the next stage.

Methyl 6-O-(3-bromobenzoyl)- $\boldsymbol{\alpha}$-D-mannopyranoside (2): Yield $80.30 \%$ as crystalline solid, M.P. $100-102{ }^{\circ} \mathrm{C}\left(\right.$ EtOAC $\left.-\mathrm{C}_{6} \mathrm{H}_{14}\right), \mathrm{R}_{f}=0.51\left(\mathrm{CH}_{3} \mathrm{OH} / \mathrm{CHCl}_{3}=1 / 8\right.$, v/v). FTIR: $v_{\max } 1685$ (-CO), 3385-3420 cm ${ }^{-1}$ (br, -OH). ${ }^{1} \mathrm{H}-\mathrm{NMR}\left(400 \mathrm{MHz}, \mathrm{CDCl}_{3}\right): \delta_{\mathrm{H}} 8.01(1 \mathrm{H}, \mathrm{s}, \mathrm{Ar}-\mathrm{H}), 7.67(1 \mathrm{H}$, $\mathrm{d}, \mathrm{J}=7.7 \mathrm{~Hz}, \operatorname{Ar}-\mathrm{H}), 7.37(1 \mathrm{H}, \mathrm{d}, \mathrm{J}=7.6 \mathrm{~Hz}, \mathrm{Ar}-\mathrm{H}), 7.21(1 \mathrm{H}, \mathrm{t}, \mathrm{J}=7.6 \mathrm{~Hz}, \operatorname{Ar}-\mathrm{H}), 5.50(1 \mathrm{H}, \mathrm{m}$, H-6a), 5.37 (1H, m, H-6b), 4.74 (1H, s, H-1), $4.18(1 \mathrm{H}, \mathrm{d}, \mathrm{J}=3.2 \mathrm{~Hz}, \mathrm{H}-2), 4.09(1 \mathrm{H}, \mathrm{t}, \mathrm{J}=9.2 \mathrm{~Hz}$, H-4), $4.00(1 \mathrm{H}, \mathrm{dd}, \mathrm{J}=3.1$ and $9.3 \mathrm{~Hz}, \mathrm{H}-3), 3.79(1 \mathrm{H}, \mathrm{m}, \mathrm{H}-5), 3.41\left(3 \mathrm{H}, \mathrm{s}, 1-\mathrm{OCH}_{3}\right)$. LC-MS $[\mathrm{M}+1]^{+} 383.90$.

Anal Calcd. for $\mathrm{C}_{14} \mathrm{H}_{23} \mathrm{O}_{7} \mathrm{Br}$ : \% C, 43.91, H, 6.04; found: \% C, 43.93, H, 6.03 .

\section{General procedure for the direct 6-O-acylation of 2,3,4-tri- $O$-acyl derivatives (3-10)}

A suspension of the 3-bromobenzoate derivative (2, $126 \mathrm{mg}, 0.18 \mathrm{mmol})$ in dry $\mathrm{N}, \mathrm{N}$ dimethylaniline $(3 \mathrm{ml})$ was cooled to $0{ }^{\circ} \mathrm{C}$ and treated with 3.3 molar equivalent ofbutyryl chloride $(0.90 \mathrm{ml})$ with continuous stirring by maintaining $0{ }^{\circ} \mathrm{C}$ for $6-7$ hours. Stirring was continued overnight at room temperature. TLC analyses showed the complete conversion of reactants into a single product. Work-up as described earlier and chromatographic purification with $\mathrm{CH}_{3} \mathrm{OH} / \mathrm{CHCl}_{3}$ mixture as eluent, afforded the butyryl derivative $\mathbf{3}(120 \mathrm{mg})$ as a crystalline solid. Recrystallization from ethyl acetatehexane gave the butyryl derivatives (3) as crystalline solid. 
Similar reaction and purification method was employed to synthesize compounds 4 (120 mg), $\mathbf{5}(110 \mathrm{mg}), \mathbf{6}(150 \mathrm{mg}), \mathbf{7}(147 \mathrm{mg}), \mathbf{8}(110 \mathrm{mg}), \mathbf{9}(100 \mathrm{mg})$, and $\mathbf{1 0}(170 \mathrm{mg})$.

Methyl 6-O-(3-bromobenzoyl)-2,3,4-tri- $\boldsymbol{O}$-butyryl- $\alpha$-D-mannopyranoside (3): Yield $84.0 \%$ as crystalline solid, M.P. $85-87{ }^{\circ} \mathrm{C}\left(\mathrm{EtOAC}-\mathrm{C}_{6} \mathrm{H}_{14}\right), \mathrm{R}_{f}=0.53\left(\mathrm{CH}_{3} \mathrm{OH} / \mathrm{CHCl}_{3}=1 / 8\right.$, v/v). FTIR: $v_{\max } 1687 \mathrm{~cm}^{-1}(-\mathrm{CO}) .{ }^{1} \mathrm{H}-\mathrm{NMR}\left(400 \mathrm{MHz}, \mathrm{CDCl}_{3}\right): \delta_{\mathrm{H}} 7.83(1 \mathrm{H}, \mathrm{s}, \mathrm{Ar}-\mathrm{H}), 7.66(1 \mathrm{H}, \mathrm{d}$, $\mathrm{J}=7.5 \mathrm{~Hz}, \mathrm{Ar}-\mathrm{H}), 7.54(1 \mathrm{H}, \mathrm{d}, \mathrm{J}=7.5 \mathrm{~Hz}, \mathrm{Ar}-\mathrm{H}), 7.33(1 \mathrm{H}, \mathrm{t}, \mathrm{J}=7.5 \mathrm{~Hz}, \mathrm{Ar}-\mathrm{H}), 5.74(1 \mathrm{H}, \mathrm{s}, \mathrm{H}-1)$, $5.68(1 \mathrm{H}, \mathrm{d}, \mathrm{J}=3.2 \mathrm{~Hz}, \mathrm{H}-2), 5.19(1 \mathrm{H}, \mathrm{dd}, \mathrm{J}=3.1$ and $9.1 \mathrm{~Hz}, \mathrm{H}-3), 4.78(1 \mathrm{H}, \mathrm{t}, \mathrm{J}=9.2 \mathrm{~Hz}, \mathrm{H}-4)$, $4.50(1 \mathrm{H}, \mathrm{m}, \mathrm{H}-6 \mathrm{a}), 4.36(1 \mathrm{H}, \mathrm{m}, \mathrm{H}-6 \mathrm{~b}), 4.19(1 \mathrm{H}, \mathrm{m}, \mathrm{H}-5), 3.42\left(3 \mathrm{H}, \mathrm{s}, 1-\mathrm{OCH}_{3}\right), 2.36\{6 \mathrm{H}, \mathrm{m}$, $\left.3 \times \mathrm{CH}_{3} \mathrm{CH}_{2} \mathrm{CH}_{2} \mathrm{CO}-\right\}, 1.77\left(6 \mathrm{H}, \mathrm{m}, 3 \times \mathrm{CH}_{3} \mathrm{CH}_{2} \mathrm{CH}_{2} \mathrm{CO}-\right), 0.99\left\{9 \mathrm{H}, \mathrm{m}, 3 \times \mathrm{CH}_{3}\left(\mathrm{CH}_{2}\right)_{2} \mathrm{CO}-\right\} . \mathrm{LC}-\mathrm{MS}$ $[\mathrm{M}+1]^{+} 593.90$.

Anal Calcd. for $\mathrm{C}_{26} \mathrm{H}_{41} \mathrm{O}_{10} \mathrm{Br}$ : \% C, 52.66, H, 6.96; found: \% C, 52.68, H, 6.97.

Methyl 6-O-(3-bromobenzoyl)-2,3,4-tri- $O$-pentanoyl- $\alpha$-D-mannopyranoside (4): Yield $84.10 \%$ as crystalline solid, M.P. $138-140{ }^{\circ} \mathrm{C}\left(\right.$ EtOAC $\left.-\mathrm{C}_{6} \mathrm{H}_{14}\right), \mathrm{R}_{f}=0.52\left(\mathrm{CH}_{3} \mathrm{OH} / \mathrm{CHCl}_{3}=1 / 8\right.$, v/v). FTIR: $v_{\max } 1697 \mathrm{~cm}^{-1}(-\mathrm{CO}) .{ }^{1} \mathrm{H}-\mathrm{NMR}\left(400 \mathrm{MHz}, \mathrm{CDCl}_{3}\right)$ : $\delta_{\mathrm{H}} 8.04(1 \mathrm{H}, \mathrm{s}, \mathrm{Ar}-\mathrm{H}), 7.86(1 \mathrm{H}, \mathrm{d}, \mathrm{J}=7.4 \mathrm{~Hz}$, Ar-H), $7.74(1 \mathrm{H}, \mathrm{d}, \mathrm{J}=7.5 \mathrm{~Hz}, \operatorname{Ar}-\mathrm{H}), 7.36(1 \mathrm{H}, \mathrm{t}, \mathrm{J}=7.4 \mathrm{~Hz}, \operatorname{Ar}-\mathrm{H}), 5.24(1 \mathrm{H}, \mathrm{s}, \mathrm{H}-1), 5.01(1 \mathrm{H}, \mathrm{d}$, $\mathrm{J}=3.3 \mathrm{~Hz}, \mathrm{H}-2), 4.81(1 \mathrm{H}, \mathrm{dd}, \mathrm{J}=3.2$ and $9.1 \mathrm{~Hz}, \mathrm{H}-3), 4.66(1 \mathrm{H}, \mathrm{t}, \mathrm{J}=9.0 \mathrm{~Hz}, \mathrm{H}-4), 4.52(1 \mathrm{H}, \mathrm{m}, \mathrm{H}-6 \mathrm{a})$, $4.16(1 \mathrm{H}, \mathrm{m}, \mathrm{H}-6 \mathrm{~b}), 4.02(1 \mathrm{H}, \mathrm{m}, \mathrm{H}-5), 3.40\left(3 \mathrm{H}, \mathrm{s}, 1-\mathrm{OCH}_{3}\right), 2.39\left\{6 \mathrm{H}, \mathrm{m}, 3 \times \mathrm{CH}_{3}\left(\mathrm{CH}_{2}\right)_{2} \mathrm{CH}_{2} \mathrm{CO}-\right\}, 1.48$ (6H, m, $\left.3 \times \mathrm{CH}_{3} \mathrm{CH}_{2} \mathrm{CH}_{2} \mathrm{CH}_{2} \mathrm{CO}-\right), 1.31\left\{6 \mathrm{H}, \mathrm{m}, 3 \times \mathrm{CH}_{3} \mathrm{CH}_{2}\left(\mathrm{CH}_{2}\right)_{2} \mathrm{CO}-\right\}, 0.91\left\{9 \mathrm{H}, \mathrm{m}, 3 \times \mathrm{CH}_{3}\left(\mathrm{CH}_{2}\right)_{3} \mathrm{CO}-\right\}$. LC-MS [M+1] $]^{+} 635.90$.

Anal Calcd. for $\mathrm{C}_{29} \mathrm{H}_{47} \mathrm{O}_{10} \mathrm{Br}$ : \% C, 54.85, H, 7.45; found: \% C, 54.87, H, 7.46.

Methyl 6-O-(3-bromobenzoyl)-2,3,4-tri- $\boldsymbol{O}$-hexanoyl- $\alpha$-D-mannopyranoside (5): Yield $90.24 \%$ as crystalline solid, M.P. $88-90{ }^{\circ} \mathrm{C}\left(\mathrm{EtOAC}-\mathrm{C}_{6} \mathrm{H}_{14}\right), \mathrm{R}_{f}=0.54\left(\mathrm{CH}_{3} \mathrm{OH} / \mathrm{CHCl}_{3}=1 / 8\right.$, v/v). FTIR: $v_{\max } 1697 \mathrm{~cm}^{-1}(-\mathrm{CO}) .{ }^{1} \mathrm{H}-\mathrm{NMR}\left(400 \mathrm{MHz}, \mathrm{CDCl}_{3}\right): \delta_{\mathrm{H}} 8.00(1 \mathrm{H}, \mathrm{s}, \mathrm{Ar}-\mathrm{H}), 7.76(1 \mathrm{H}, \mathrm{d}, \mathrm{J}=7.5 \mathrm{~Hz}$, Ar-H), $7.72(1 \mathrm{H}, \mathrm{d}, \mathrm{J}=7.5 \mathrm{~Hz}, \operatorname{Ar}-\mathrm{H}), 7.28(1 \mathrm{H}, \mathrm{t}, \mathrm{J}=7.4 \mathrm{~Hz}, \mathrm{Ar}-\mathrm{H}), 5.20(1 \mathrm{H}, \mathrm{s}, \mathrm{H}-1), 5.14(1 \mathrm{H}, \mathrm{d}$, $\mathrm{J}=3.3 \mathrm{~Hz}, \mathrm{H}-2), 4.88(1 \mathrm{H}, \mathrm{dd}, \mathrm{J}=3.2$ and $9.1 \mathrm{~Hz}, \mathrm{H}-3), 4.60(1 \mathrm{H}, \mathrm{t}, \mathrm{J}=9.0 \mathrm{~Hz}, \mathrm{H}-4), 4.41$ (1H, m, H-6a), $4.36(1 \mathrm{H}, \mathrm{m}, \mathrm{H}-6 \mathrm{~b}), 4.11(1 \mathrm{H}, \mathrm{m}, \mathrm{H}-5), 3.42\left(3 \mathrm{H}, \mathrm{s}, 1-\mathrm{OCH}_{3}\right), 2.36\left\{6 \mathrm{H}, \mathrm{m}, 3 \times \mathrm{CH}_{3}\left(\mathrm{CH}_{2}\right)_{3} \mathrm{CH}_{2} \mathrm{CO}-\right\}$, $1.61\left\{6 \mathrm{H}, \mathrm{m}, 3 \times \mathrm{CH}_{3}\left(\mathrm{CH}_{2}\right)_{2} \mathrm{CH}_{2} \mathrm{CH}_{2} \mathrm{CO}-\right\}, 1.25\left\{12 \mathrm{H}, \mathrm{m}, 3 \times \mathrm{CH}_{3}\left(\mathrm{CH}_{2}\right)_{2} \mathrm{CH}_{2} \mathrm{CH}_{2} \mathrm{CO}-\right\}, 0.89\{9 \mathrm{H}, \mathrm{m}$, $\left.3 \times \mathrm{CH}_{3}\left(\mathrm{CH}_{2}\right)_{4} \mathrm{CO}-\right\}$. LC-MS [M+1] 677.90 .

Anal Calcd. for $\mathrm{C}_{32} \mathrm{H}_{53} \mathrm{O}_{10} \mathrm{Br}$ : \% C, 56.77, $\mathrm{H}, 7.88$; found: \% C, 56.76, H, 7.89.

Methyl 6-O-(3-bromobenzoyl)-2,3,4-tri- $\boldsymbol{O}$-heptanoyl- $\alpha$-D-mannopyranoside(6): Yield $71.31 \%$ as crystalline solid, M.P. $108-110{ }^{\circ} \mathrm{C}\left(\mathrm{EtOAC}-\mathrm{C}_{6} \mathrm{H}_{14}\right), \mathrm{R}_{f}=0.51\left(\mathrm{CH}_{3} \mathrm{OH} / \mathrm{CHCl}_{3}=1 / 9\right.$, v/v). FTIR: $v_{\max } 1685 \mathrm{~cm}^{-1}(-\mathrm{CO}) .{ }^{1} \mathrm{H}-\mathrm{NMR}\left(400 \mathrm{MHz}, \mathrm{CDCl}_{3}\right): \delta_{\mathrm{H}} 8.05(1 \mathrm{H}, \mathrm{s}, \mathrm{Ar}-\mathrm{H}), 7.74(1 \mathrm{H}, \mathrm{d}, \mathrm{J}=7.6 \mathrm{~Hz}$, Ar-H), $7.54(1 \mathrm{H}, \mathrm{d}, \mathrm{J}=7.6 \mathrm{~Hz}, \mathrm{Ar}-\mathrm{H}), 7.30(1 \mathrm{H}, \mathrm{t}, \mathrm{J}=7.6 \mathrm{~Hz}, \mathrm{Ar}-\mathrm{H}), 4.89(1 \mathrm{H}, \mathrm{s}, \mathrm{H}-1), 4.74(1 \mathrm{H}, \mathrm{d}$, $\mathrm{J}=3.5 \mathrm{~Hz}, \mathrm{H}-2), 4.71(1 \mathrm{H}, \mathrm{dd}, \mathrm{J}=3.3$ and $9.0 \mathrm{~Hz}, \mathrm{H}-3), 4.66(1 \mathrm{H}, \mathrm{t}, \mathrm{J}=9.1 \mathrm{~Hz}, \mathrm{H}-4), 4.40$ (1H, m, H-6a), $4.35(1 \mathrm{H}, \mathrm{m}, \mathrm{H}-6 \mathrm{~b}), 4.10(1 \mathrm{H}, \mathrm{m}, \mathrm{H}-5), 3.44\left(3 \mathrm{H}, \mathrm{s}, 1-\mathrm{OCH}_{3}\right), 2.36\left\{6 \mathrm{H}, \mathrm{m}, 3 \times \mathrm{CH}_{3}\left(\mathrm{CH}_{2}\right)_{4} \mathrm{CH}_{2} \mathrm{CO}-\right\}$, $1.68\left\{6 \mathrm{H}, \mathrm{m}, 3 \times \mathrm{CH}_{3}\left(\mathrm{CH}_{2}\right)_{3} \mathrm{CH}_{2} \mathrm{CH}_{2} \mathrm{CO}-\right\}, 1.38\left\{18 \mathrm{H}, \mathrm{m}, 3 \times \mathrm{CH}_{3}\left(\mathrm{CH}_{2}\right)_{3} \mathrm{CH}_{2} \mathrm{CH}_{2} \mathrm{CO}-\right\}, 0.89\{9 \mathrm{H}, \mathrm{m}$, $\left.3 \times \mathrm{CH}_{3}\left(\mathrm{CH}_{2}\right)_{5} \mathrm{CO}-\right\}$. LC-MS [M+1] $]^{+} 719.90$.

Anal Calcd. for $\mathrm{C}_{35} \mathrm{H}_{59} \mathrm{O}_{10} \mathrm{Br}$ : \% C, 58.47, H, 8.26; found: \% C, 58.46, H, 8.28\%.

Methyl 6- $\boldsymbol{O}$-(3-bromobenzoyl)-2,3,4-tri- $\boldsymbol{O}$-octanoyl- $\boldsymbol{\alpha}$-D-mannopyranoside (7): Yield $90.11 \%$ as crystalline solid, M.P. $121-123{ }^{\circ} \mathrm{C}\left(\right.$ EtOAC $\left.-\mathrm{C}_{6} \mathrm{H}_{14}\right), \mathrm{R}_{f}=0.55\left(\mathrm{CH}_{3} \mathrm{OH} / \mathrm{CHCl}_{3}=1 / 8\right.$, v/v). FTIR: $v_{\max }$ $1698 \mathrm{~cm}^{-1}(-\mathrm{CO}) .{ }^{1} \mathrm{H}-\mathrm{NMR}\left(400 \mathrm{MHz}, \mathrm{CDCl}_{3}\right): \delta_{\mathrm{H}} 8.04(1 \mathrm{H}, \mathrm{s}, \mathrm{Ar}-\mathrm{H}), 7.67(1 \mathrm{H}, \mathrm{d}, \mathrm{J}=7.7 \mathrm{~Hz}, \mathrm{Ar}-\mathrm{H})$, $7.53(1 \mathrm{H}, \mathrm{d}, \mathrm{J}=7.6 \mathrm{~Hz}, \mathrm{Ar}-\mathrm{H}), 7.33(1 \mathrm{H}, \mathrm{t}, \mathrm{J}=7.6 \mathrm{~Hz}, \mathrm{Ar}-\mathrm{H}), 5.09(1 \mathrm{H}, \mathrm{s}, \mathrm{H}-1), 5.04(1 \mathrm{H}, \mathrm{d}, \mathrm{J}=3.5 \mathrm{~Hz}$, 
H-2), $4.88(1 \mathrm{H}, \mathrm{dd}, \mathrm{J}=3.3$ and $9.0 \mathrm{~Hz}, \mathrm{H}-3), 4.79(1 \mathrm{H}, \mathrm{t}, \mathrm{J}=9.1 \mathrm{~Hz}, \mathrm{H}-4), 4.38(1 \mathrm{H}, \mathrm{m}, \mathrm{H}-6 \mathrm{a}), 4.31(1 \mathrm{H}$, m, H-6b), $4.00(1 \mathrm{H}, \mathrm{m}, \mathrm{H}-5), 3.42\left(3 \mathrm{H}, \mathrm{s}, 1-\mathrm{OCH}_{3}\right), 2.36\left\{6 \mathrm{H}, \mathrm{m}, 3 \times \mathrm{CH}_{3}\left(\mathrm{CH}_{2}\right)_{5} \mathrm{CH} \mathrm{C}_{2} \mathrm{CO}-\right\}, 1.71\{6 \mathrm{H}, \mathrm{m}$, $\left.3 \times \mathrm{CH}_{3}\left(\mathrm{CH}_{2}\right)_{4} \mathrm{CH}_{2} \mathrm{CH}_{2} \mathrm{CO}-\right\}, 1.29\left\{24 \mathrm{H}, \mathrm{m}, 3 \times \mathrm{CH}_{3}\left(\mathrm{CH}_{2}\right)_{4}\left(\mathrm{CH}_{2}\right)_{2} \mathrm{CO}-\right\}, 0.89\left\{9 \mathrm{H}, \mathrm{m}, 3 \times \mathrm{CH}_{3}\left(\mathrm{CH}_{2}\right)_{6} \mathrm{CO}-\right\}$. LC-MS $[\mathrm{M}+1]^{+} 761.90$.

Anal Calcd. for $\mathrm{C}_{38} \mathrm{H}_{65} \mathrm{O}_{10} \mathrm{Br}$ : \% C, 59.98, H, 8.60; found: \% C, 59.99, H, 8.62.

Methyl 6- $\boldsymbol{O}$-(3-bromobenzoyl)-2,3,4-tri- $\boldsymbol{O}$-lauroyl- $\alpha$-D-mannopyranoside (8): Yield $80.24 \%$ as crystalline solid, M.P. $109-111{ }^{\circ} \mathrm{C}\left(\right.$ EtOAC $\left.-\mathrm{C}_{6} \mathrm{H}_{14}\right), \mathrm{R}_{f}=0.52\left(\mathrm{CH}_{3} \mathrm{OH} / \mathrm{CHCl}_{3}=1 / 8\right.$, v/v). FTIR: $v_{\max } 1688 \mathrm{~cm}^{-1}$ (-CO). ${ }^{1} \mathrm{H}-\mathrm{NMR}\left(400 \mathrm{MHz}, \mathrm{CDCl}_{3}\right): \delta_{\mathrm{H}} 8.01(1 \mathrm{H}, \mathrm{s}, \mathrm{Ar}-\mathrm{H}), 7.78(1 \mathrm{H}, \mathrm{d}, \mathrm{J}=7.5 \mathrm{~Hz}$, Ar-H), $7.59(1 \mathrm{H}, \mathrm{d}, \mathrm{J}=7.5 \mathrm{~Hz}, \mathrm{Ar}-\mathrm{H}), 7.41(1 \mathrm{H}, \mathrm{t}, \mathrm{J}=7.5 \mathrm{~Hz}, \mathrm{Ar}-\mathrm{H}), 5.11(1 \mathrm{H}, \mathrm{s}, \mathrm{H}-1), 5.07(1 \mathrm{H}, \mathrm{d}$, $\mathrm{J}=3.6 \mathrm{~Hz}, \mathrm{H}-2), 4.91(1 \mathrm{H}, \mathrm{dd}, \mathrm{J}=3.3$ and $9.0 \mathrm{~Hz}, \mathrm{H}-3), 4.71(1 \mathrm{H}, \mathrm{t}, \mathrm{J}=9.2 \mathrm{~Hz}, \mathrm{H}-4), 4.35(1 \mathrm{H}, \mathrm{m}, \mathrm{H}-6 \mathrm{a})$, $4.30(1 \mathrm{H}, \mathrm{m}, \mathrm{H}-6 \mathrm{~b}), 4.07(1 \mathrm{H}, \mathrm{m}, \mathrm{H}-5), 3.41\left(3 \mathrm{H}, \mathrm{s}, 1-\mathrm{OCH}_{3}\right), 2.37\left\{6 \mathrm{H}, \mathrm{m}, 3 \times \mathrm{CH}_{3}\left(\mathrm{CH}_{2}\right)_{9} \mathrm{CH}_{2} \mathrm{CO}-\right\}$, $1.66\left\{6 \mathrm{H}, \mathrm{m}, 3 \times \mathrm{CH}_{3}\left(\mathrm{CH}_{2}\right)_{8} \mathrm{CH}_{2} \mathrm{CH}_{2} \mathrm{CO}\right\}, 1.27\left\{48 \mathrm{H}, \mathrm{m}, 3 \times \mathrm{CH}_{3}\left(\mathrm{CH}_{2}\right)_{8} \mathrm{CH}_{2} \mathrm{CH}_{2} \mathrm{CO}-\right\}, 0.91\{9 \mathrm{H}, \mathrm{m}$, $\left.3 \times \mathrm{CH}_{3}\left(\mathrm{CH}_{2}\right)_{10} \mathrm{CO}-\right\}$. LC-MS $[\mathrm{M}+1]^{+} 929.90$.

Anal Calcd. for $\mathrm{C}_{50} \mathrm{H}_{89} \mathrm{O}_{10} \mathrm{Br}$ : \% C, 64.65, H, 9.65; found: \% C, 64.67, H, 9.66.

Methyl 6-O-(3-bromobenzoyl)-2,3,4-tri- $\boldsymbol{O}$-palmitoyl- $\alpha$-D-mannopyranoside (9): Yield $78.47 \%$ as crystalline solid, M.P. $100-102{ }^{\circ} \mathrm{C}\left(\right.$ EtOAC $\left.-\mathrm{C}_{6} \mathrm{H}_{14}\right), \mathrm{R}_{f}=0.53\left(\mathrm{CH}_{3} \mathrm{OH} / \mathrm{CHCl}_{3}=1 / 9\right.$, v/v). FTIR: $v_{\max }$ $1697 \mathrm{~cm}^{-1}(-\mathrm{CO}) .{ }^{1} \mathrm{H}-\mathrm{NMR}\left(400 \mathrm{MHz}, \mathrm{CDCl}_{3}\right): \delta_{\mathrm{H}} 7.88(1 \mathrm{H}, \mathrm{s}, \mathrm{Ar}-\mathrm{H}), 7.71(1 \mathrm{H}, \mathrm{d}, \mathrm{J}=7.4 \mathrm{~Hz}, \mathrm{Ar}-\mathrm{H})$, $7.66(1 \mathrm{H}, \mathrm{d}, \mathrm{J}=7.5 \mathrm{~Hz}, \mathrm{Ar}-\mathrm{H}), 7.40(1 \mathrm{H}, \mathrm{t}, \mathrm{J}=7.6 \mathrm{~Hz}, \mathrm{Ar}-\mathrm{H}), 5.06(1 \mathrm{H}, \mathrm{s}, \mathrm{H}-1), 4.66(1 \mathrm{H}, \mathrm{d}, \mathrm{J}=3.5 \mathrm{~Hz}$, H-2), $4.51(1 \mathrm{H}, \mathrm{dd}, \mathrm{J}=3.2$ and $9.1 \mathrm{~Hz}, \mathrm{H}-3), 4.41(1 \mathrm{H}, \mathrm{t}, \mathrm{J}=9.1 \mathrm{~Hz}, \mathrm{H}-4), 3.85(1 \mathrm{H}, \mathrm{m}, \mathrm{H}-6 \mathrm{a}), 3.70$ $(1 \mathrm{H}, \mathrm{m}, \mathrm{H}-6 \mathrm{~b}), 3.67(1 \mathrm{H}, \mathrm{m}, \mathrm{H}-5), 3.42\left(3 \mathrm{H}, \mathrm{s}, 1-\mathrm{OCH}_{3}\right), 2.36\left\{6 \mathrm{H}, \mathrm{m}, 3 \times \mathrm{CH}_{3}\left(\mathrm{CH}_{2}\right)_{13} \mathrm{CH}_{2} \mathrm{CO}-\right\}, 1.69$ $\left\{78 \mathrm{H}, \mathrm{m}, 3 \times \mathrm{CH}_{3}\left(\mathrm{CH}_{2}\right)_{13} \mathrm{CH}_{2} \mathrm{CO}-\right\}, 1.26\left\{9 \mathrm{H}, \mathrm{s},\left(\mathrm{CH}_{3}\right)_{3} \mathrm{CCO}-\right\}, 0.90\left\{9 \mathrm{H}, \mathrm{m}, 3 \times \mathrm{CH}_{3}\left(\mathrm{CH}_{2}\right)_{14} \mathrm{CO}-\right\} . \mathrm{LC}-\mathrm{MS}$ $[\mathrm{M}+1]^{+} 1097.90$.

Anal Calcd. for $\mathrm{C}_{62} \mathrm{H}_{113} \mathrm{O}_{10} \mathrm{Br}$ : \% C, 67.88, H, 10.37; found: \% C, 67.87, H, 10.39.

Methyl 6-O-(3-bromobenzoyl)-2,3,4-tri- $\boldsymbol{O}$-trityl- $\alpha$-D-mannopyranoside (10): Yield $88.15 \%$ as crystalline solid, M.P. $111-113{ }^{\circ} \mathrm{C}\left(\mathrm{EtOAC}-\mathrm{C}_{6} \mathrm{H}_{14}\right), \mathrm{R}_{f}=0.55\left(\mathrm{CH}_{3} \mathrm{OH} / \mathrm{CHCl}_{3}=1 / 9\right.$, v/v). FTIR: $v_{\max }$ $1692 \mathrm{~cm}^{-1}(-\mathrm{CO}) .{ }^{1} \mathrm{H}-\mathrm{NMR}\left(400 \mathrm{MHz}, \mathrm{CDCl}_{3}\right): \delta_{\mathrm{H}} 8.05(1 \mathrm{H}, \mathrm{s}, \mathrm{Ar}-\mathrm{H}), 7.85(1 \mathrm{H}, \mathrm{d}, \mathrm{J}=7.7 \mathrm{~Hz}, \mathrm{Ar}-\mathrm{H})$, $7.66(1 \mathrm{H}, \mathrm{d}, \mathrm{J}=7.6 \mathrm{~Hz}, \mathrm{Ar}-\mathrm{H}), 7.36(18 \mathrm{H}, \mathrm{m}, 3 \times \mathrm{Ar}-\mathrm{H}), 7.33(27 \mathrm{H}, \mathrm{m}, 3 \times \mathrm{Ar}-\mathrm{H}), 7.31(1 \mathrm{H}, \mathrm{t}, \mathrm{J}=7.5 \mathrm{~Hz}$, Ar -H), $5.23(1 \mathrm{H}, \mathrm{s}, \mathrm{H}-1), 5.00(1 \mathrm{H}, \mathrm{d}, \mathrm{J}=3.7 \mathrm{~Hz}, \mathrm{H}-2), 4.90(1 \mathrm{H}, \mathrm{dd}, \mathrm{J}=3.5$ and $9.2 \mathrm{~Hz}, \mathrm{H}-3), 4.61(1 \mathrm{H}$, t, J = 9.2 Hz, H-4), 4.25 (1H, m, H-6a), 4.22 (1H, m, H-6b), 4.16 (1H, m, H-5), 3.43 (3H, s, 1-OCH $)$. LC-MS $[\mathrm{M}+1]^{+} 1109.90$.

Anal Calcd. for $\mathrm{C}_{71} \mathrm{H}_{65} \mathrm{O}_{7} \mathrm{Br}$ : \% C, 76.90, H, 5.90; found: \% C, 76.92, H, 5.91 .

\section{X-ray powder diffraction}

The single-crystal X-ray diffraction method is mainly used for structure determination while the X-ray powder diffraction method is mainly used for quantitative identification of crystalline compounds. The diffraction pattern of crystal structure also provides information on determining the dimension of the unit cell of the crystal lattice and the atomic arrangement within the cell. X-ray powder diffraction was performed using Rigaku Dmax2200PC diffractometer (Rigaku Corporation, Tokyo, Japan) and $\mathrm{Cu} \mathrm{K \alpha}$-radiation ( $\chi=1.54060 \mathrm{~A}^{\circ}$, intensity range $\left.5^{\circ} \leq 2 \theta \leq 90^{\circ}\right)$ at $40 \mathrm{KeV}$ and $40 \mathrm{~mA}$ and step length of $0.06^{\circ}$ with step time $1 \mathrm{~s}$ in the scan range of $2 \theta$ from $0^{\circ}-50^{\circ}$ [24]. By using Bragg's law, the interlayer $\mathrm{d}$-spacing was calculated. If $\mathrm{h}, \mathrm{k}$, and 1 represent the miller indices, the rules of the determination of crystal lattice type are as follows (Table 1). 
Table 1. Rules of the determination of crystal lattice type

\begin{tabular}{|c|c|}
\hline Lattice type & Rules for reflection to be observed \\
\hline Primitive, $\mathrm{P}$ & None \\
\hline Body centered, I & $\mathrm{hkl} ; \mathrm{h}+\mathrm{k}+\mathrm{l}=2 \mathrm{n}$ \\
\hline Face centered, F & $\mathrm{hkl} ; \mathrm{h}, \mathrm{k}, 1$ either all odd or all even \\
\hline Side centered, C & $\mathrm{hkl} ; \mathrm{h}+\mathrm{k}=2 \mathrm{n}$ \\
\hline Rhombohedral & $\mathrm{hkl} ;-\mathrm{h}+\mathrm{k}+\mathrm{l}=3 \mathrm{n}$ or $\mathrm{h}-\mathrm{k}+\mathrm{l}=3 \mathrm{n}$ \\
\hline
\end{tabular}

\section{Results and discussion}

\section{Chemistry}

The present work reported here was to study regioselective 3-bromobenzoylation ofmethyl $\alpha$-Dmannopyranoside (1) using the direct method (Fig. 2). The resulting 3-bromobenzoylation products were converted to a number of derivatives using a series of acylating agents e.g., butyryl chloride, pentanoyl chloride, hexanoyl chloride, heptanoyl chloride, octanoyl chloride, lauroylchloride, palmitoyl chloride and trityl chloride (Table 2).

\section{Characterization and selective 3-bromobenzoylation \\ of mannopyranoside}

Our initial effort was to treatment of methyl $\alpha$-D-mannopyranoside (1) with 3-bromobenzoyl chloride as an acylating agent in dry DMF at $-5{ }^{\circ} \mathrm{C}$ and after usual work-up, compound 2 was obtained in good yields. This compound was sufficiently pure for use in the next stages. However,

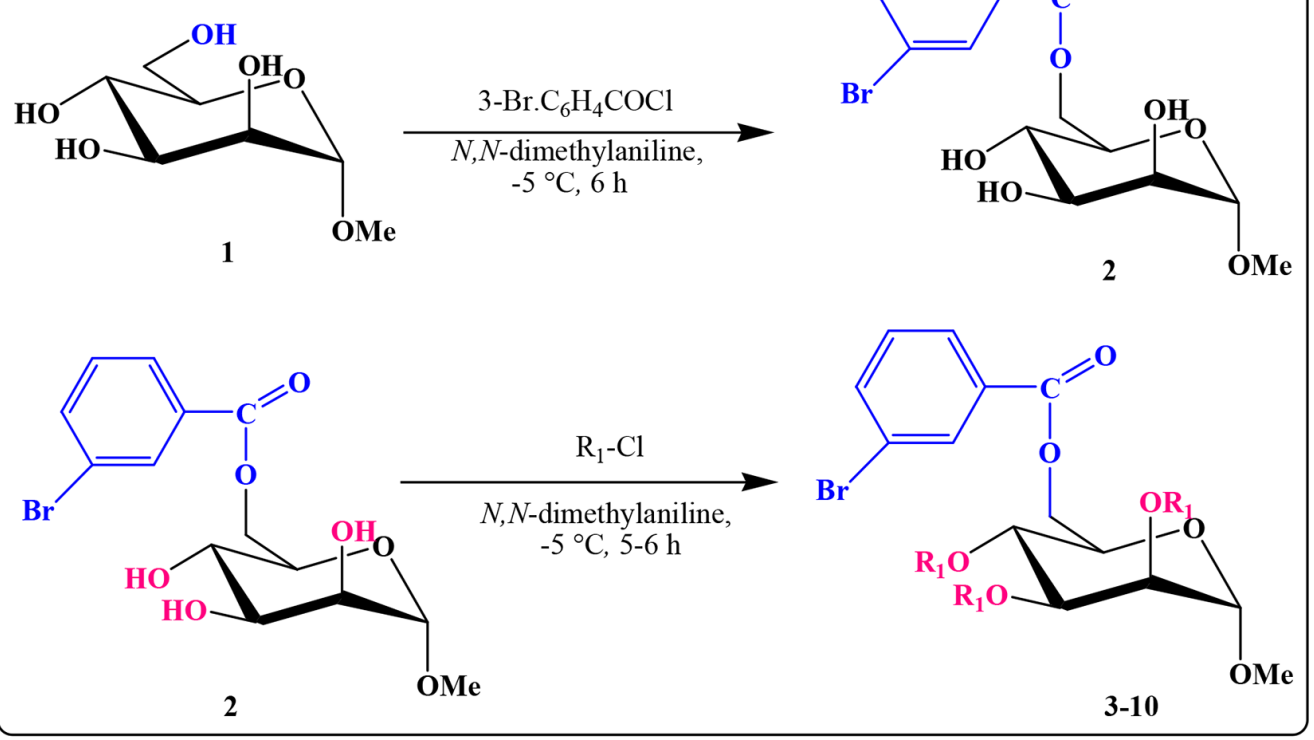

Fig. 2. Reagents and conditions: dry $\operatorname{HCON}\left(\mathrm{CH}_{3}\right)_{2},-5^{\circ} \mathrm{C}$, DMAP, stirrer for $5-6 \mathrm{~h}, \mathrm{R}_{1}=$ several acyl halides $(\mathbf{3}-\mathbf{1 0})$ 
Table 2. List of the synthesized compounds of methyl $\alpha$-D-mannopyranoside derivatives (2-10)

\begin{tabular}{|c|c|c|}
\hline Entry & Acyl group (6-OH) & Acyl group $\left(\mathbf{R}_{1}\right)$ \\
\hline 1 & -- & -- \\
\hline 2 & $3-\mathrm{Br} . \mathrm{C}_{6} \mathrm{H}_{4} \mathrm{CO}-$ & -- \\
\hline 3 & $3-\mathrm{Br} . \mathrm{C}_{6} \mathrm{H}_{4} \mathrm{CO}-$ & $\mathrm{CH}_{3}\left(\mathrm{CH}_{2}\right)_{2} \mathrm{CO}-$ \\
\hline 4 & $3-\mathrm{Br} . \mathrm{C}_{6} \mathrm{H}_{4} \mathrm{CO}-$ & $\mathrm{CH}_{3}\left(\mathrm{CH}_{2}\right)_{3} \mathrm{CO}-$ \\
\hline 5 & $3-\mathrm{Br} . \mathrm{C}_{6} \mathrm{H}_{4} \mathrm{CO}-$ & $\mathrm{CH}_{3}\left(\mathrm{CH}_{2}\right)_{4} \mathrm{CO}-$ \\
\hline 6 & $3-\mathrm{Br} \cdot \mathrm{C}_{6} \mathrm{H}_{4} \mathrm{CO}-$ & $\mathrm{CH}_{3}\left(\mathrm{CH}_{2}\right)_{5} \mathrm{CO}-$ \\
\hline 7 & $3-\mathrm{Br} \cdot \mathrm{C}_{6} \mathrm{H}_{4} \mathrm{CO}-$ & $\mathrm{CH}_{3}\left(\mathrm{CH}_{2}\right)_{6} \mathrm{CO}-$ \\
\hline 8 & $3-\mathrm{Br} \cdot \mathrm{C}_{6} \mathrm{H}_{4} \mathrm{CO}-$ & $\mathrm{CH}_{3}\left(\mathrm{CH}_{2}\right)_{10} \mathrm{CO}-$ \\
\hline 9 & $3-\mathrm{Br} . \mathrm{C}_{6} \mathrm{H}_{4} \mathrm{CO}-$ & $\mathrm{CH}_{3}\left(\mathrm{CH}_{2}\right)_{14} \mathrm{CO}-$ \\
\hline 10 & $3-\mathrm{Br} . \mathrm{C}_{6} \mathrm{H}_{4} \mathrm{CO}-$ & $\mathrm{Ph}_{3} \mathrm{CO}_{-}$ \\
\hline
\end{tabular}
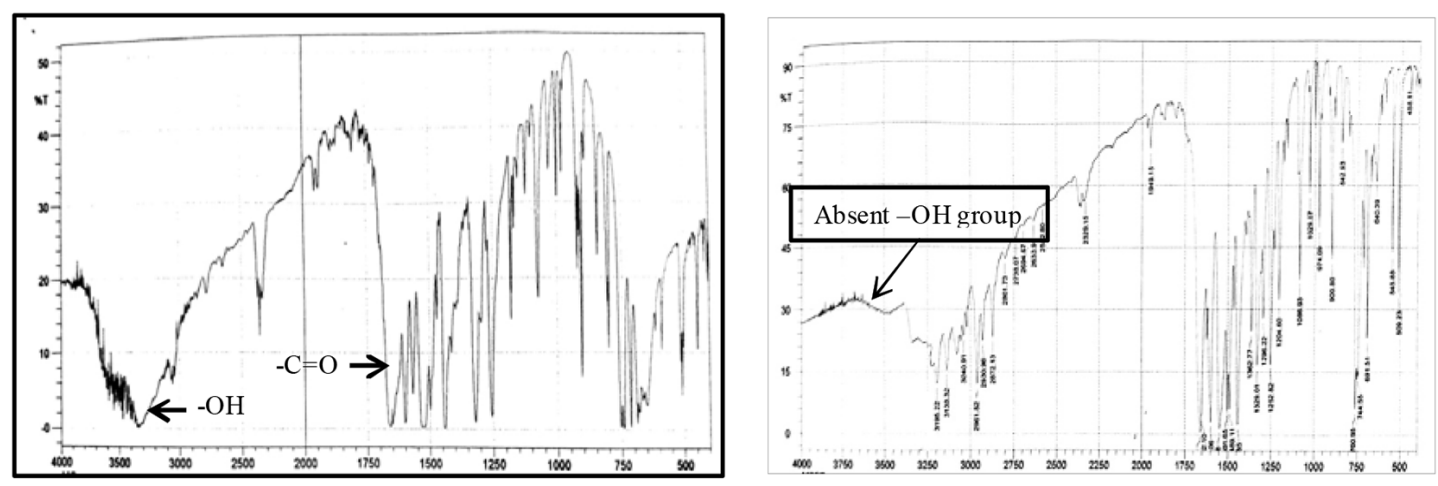

Fig. 3. IR spectrum of the compounds $\mathbf{2}$ (right) and $\mathbf{3}$ (left)

an analytical sample was prepared by recrystallization from ethyl acetate-hexane. The IR spectrum of compound 2 showed absorption bands at $1685 \mathrm{~cm}^{-1}$ (-CO stretching) and 3385-3420 $\mathrm{cm}^{-1}$ (br, -OH) (-OH stretching), thereby suggesting the presence of carbonyl and hydroxyl groups in the molecule (Fig. 3). In its ${ }^{1} \mathrm{H}-\mathrm{NMR}$ spectrum the one-proton singlet at $\delta 8.01(\mathrm{Ar}-\mathrm{H})$, two one-proton doublets at $\delta$ $7.67(\mathrm{~J} 7.7 \mathrm{~Hz}$ ) and $\delta 7.37(\mathrm{~J} 7.6 \mathrm{~Hz}$ ), and one-proton triplet at $\delta 7.21$ (J 7.6 Hz, Ar-H) corresponded to the aromatic protons inthe 3-bromobenzoyl group (Fig. 4). The large downfield shift of C-6 protons to $\delta 5.50$ (as m, H-6a) and $\delta 5.37$ (as $\mathrm{m}, \mathrm{H}-6 \mathrm{~b}$ ) from their precursor (1) values and the resonances of other protons in their anticipated positions showed the attachment of 3-bromobenzoyl group less hindered and more reactive position at 6 . Further support for the structure of compound $\mathbf{2}$ was achieved from its mass spectrum which displayed the molecular ion peak at $m / z[\mathbf{M}+1]^{+} 383.90$ that corresponded to the molecular formula of $\mathrm{C}_{14} \mathrm{H}_{23} \mathrm{O}_{7} \mathrm{Br}$. Complete analysis of the IR, ${ }^{1} \mathrm{H}-\mathrm{NMR}$, and mass spectra of this compound was in agreement withmethyl 6-O-(3-bromobenzoyl)- $\alpha$-D-mannopyranoside (2). This result is similar to that observed by Kawsar et al. [25].

The structure of compound 2 was supported by the preparation of its butyryl derivative 3 . IR spectrum showed only absorption band at $1687 \mathrm{~cm}^{-1}$ for $-\mathrm{CO}$ stretching but there is no $-\mathrm{OH}$ stretching band (Fig. 3). As expected the ${ }^{1}$ H-NMR spectrumof this compound contained characteristics of two 


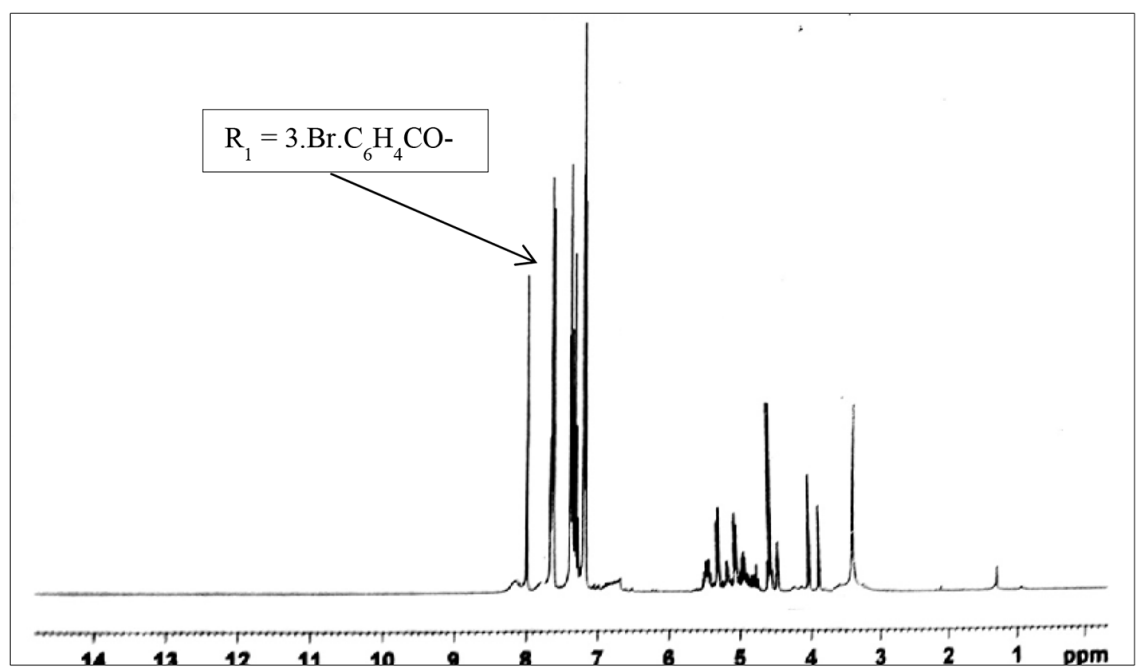

Fig. 4. ${ }^{1} \mathrm{H}-\mathrm{NMR}$ spectra of the compound 2

six-proton multiplets at $\delta 2.36\left\{3 \times \mathrm{CH}_{3} \mathrm{CH}_{2} \mathrm{CH}_{2} \mathrm{CO}-\right\}$ and $\delta 1.77\left\{3 \times \mathrm{CH}_{3} \mathrm{CH}_{2} \mathrm{CH}_{2} \mathrm{CO}-\right\}$ and nine-proton multiplet at $\delta 0.99\left\{3 \times \mathrm{CH}_{3}\left(\mathrm{CH}_{2}\right)_{2} \mathrm{CO}-\right\}$ corresponding to the one butyryl group. The downfield shifts of C-2 ( $\delta 5.68, \mathrm{~d}, \mathrm{~J} 3.2 \mathrm{~Hz}, \mathrm{H}-2), \mathrm{C}-3$ ( $\delta 5.19$, dd, J 3.1 and $9.1 \mathrm{~Hz}, \mathrm{H}-3)$, and C-4 ( $\delta$ 4.78, t, J 9.2 Hz, H-4), as compared to the precursor triol $2(\delta 3.72 ; \delta 4.10 ; \delta 4.18)$, indicated the attachment of the three butyryl groups at positions 2, 3 and 4. The mass spectrum of compound 3 contained a molecular ion peak at $\mathrm{m} / \mathrm{z}$ $[\mathrm{M}+1]^{+} 593.90$ that corresponded to the same molecular formula, $\mathrm{C}_{26} \mathrm{H}_{41} \mathrm{O}_{10} \mathrm{Br}$. By complete analysis of the IR, ${ }^{1} \mathrm{H}-\mathrm{NMR}$ and massspectra, the structure of the tributyrate was ascertained as methyl 6-O-(3bromobenzoyl)-2,3,4-tri- $O$-butyryl- $\alpha$-D-mannopyranoside (3). The structure of 3-bromobenzoyl derivative 2 was confirmed by preparing its pentanoyl derivative 4 with pentanoyl chloride.

Additional support for the structure accorded to compound (2) was obtained by its conversion to its hexanoyl derivative (5). Thus, the reaction of compound $\mathbf{2}$ with hexanoyl chloride at freezing temperature, furnished the compound (5) in excellent yields, 90.24\%. The ${ }^{1} \mathrm{H}-\mathrm{NMR}$ spectrum of the compound 5 displayed two six-proton multiplets at $\delta 2.36\left\{3 \times \mathrm{CH}_{3}\left(\mathrm{CH}_{2}\right)_{3} \mathrm{CH}_{2} \mathrm{CO}-\right\}$, and $\delta 1.61$ $\left\{3 \times\left(\mathrm{CH}_{3}\right)_{2} \mathrm{CH}_{2} \mathrm{CH}_{2} \mathrm{CO}-\right\}$, twelve-proton multiplet at $\delta 1.25\left\{3 \times \mathrm{CH}_{3}\left(\mathrm{CH}_{2}\right)_{2} \mathrm{CH}_{2} \mathrm{CH}_{2} \mathrm{CO}-\right\}$ and nineproton multiplet at $\delta 0.89\left\{3 \times \mathrm{CH}_{3}\left(\mathrm{CH}_{2}\right)_{4} \mathrm{CO}-\right\}$ showing the attachment of three hexanoyl groups in the molecule. The resonance for $\mathrm{C}-2, \mathrm{C}-3$ and $\mathrm{C}-4$ appeared at $\delta 5.14, \delta 4.88$ and $\delta 4.60$ which shifted downfield from their precursor values indicating the presence of the three hexanoyl groups. Complete analysis of all spectra enabled us to propose the structure of this compound as methyl 6-O-(3-bromobenzoyl)-2,3,4-tri- $O$-hexanoyl- $\alpha$-D-mannopyranoside (5). As same as the structure accorded to compound $\mathbf{2}$ was finally confirmed by transformation and identification of its heptanoy $\mathbf{1 6}$, octanoy 17 , lauroy 18 , and palmitoy 19 derivatives and these structures were established by analysis of their spectroscopic data.

Finally, tritylation was carried out with an excess of trityl chlorideand isolated title derivative (10). In its ${ }^{1} \mathrm{H}-\mathrm{NMR}$ spectrum two characteristic peaks; eighteen-proton multiplet at $\delta 7.36(3 \times \mathrm{Ar}-\mathrm{H})$ and a twenty-seven-proton multiplet at $\delta 7.33(3 \times \mathrm{Ar}-\mathrm{H})$ was due to the three trityl groups in the molecule. The rest of the protons resonated in their anticipated positions and this led us to propose a structure 
of this compound as methyl 6-O-(3-bromobenzoyl)-2,3,4-tri- $O$-trityl- $\alpha$-D-mannopyranoside (10). The synthesis was found to be very promising since in all the cases, a single, mono-substitution product was isolated in reasonably high yields. These newly synthesized products may be used as important precursors for the modification of the mannopyranoside molecule at different positions.

\section{XRD measurements}

Crystallographic structures of the synthesized compounds $(7,8$ and 10) were evaluated by the $\mathrm{X}$-ray powder diffraction at room temperature. All the compounds 7, 8 and $\mathbf{1 0}$ showed many lines with high intensity in their X-ray diffraction pattern (Fig. 5 and 6) which indicates that all the compounds are well crystalline. The XRD pattern of the synthesis compounds ispresented in Table 3.

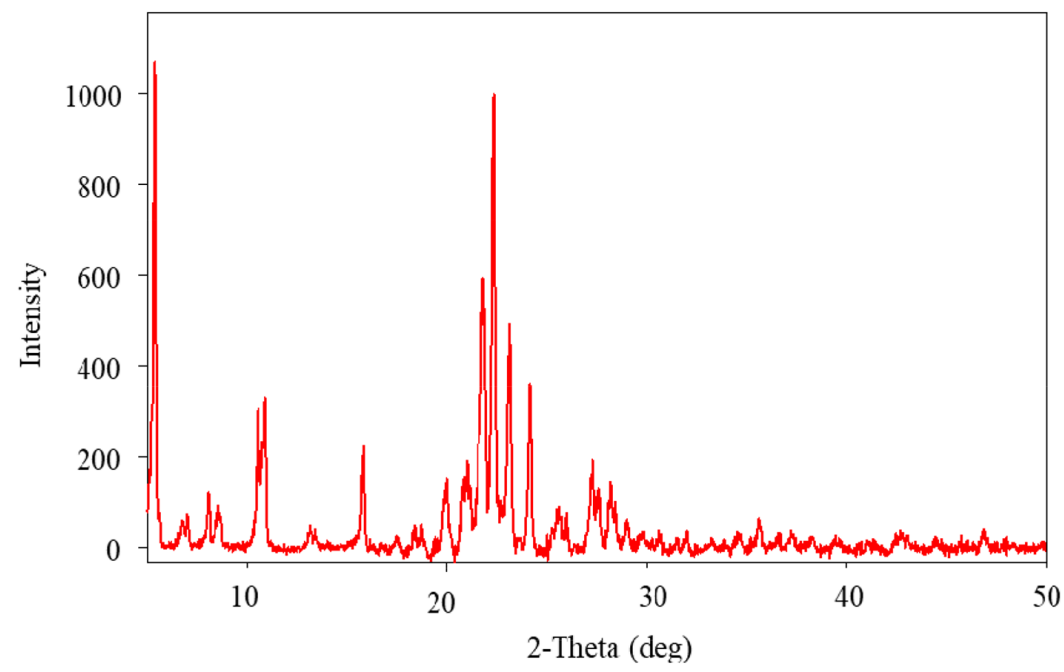

Fig. 5. XRD pattern of methyl 6-O-(3-bromobenzoyl)-2,3,4-tri- $O$-octanoyl- $\alpha$-D-mannopyranoside (7)

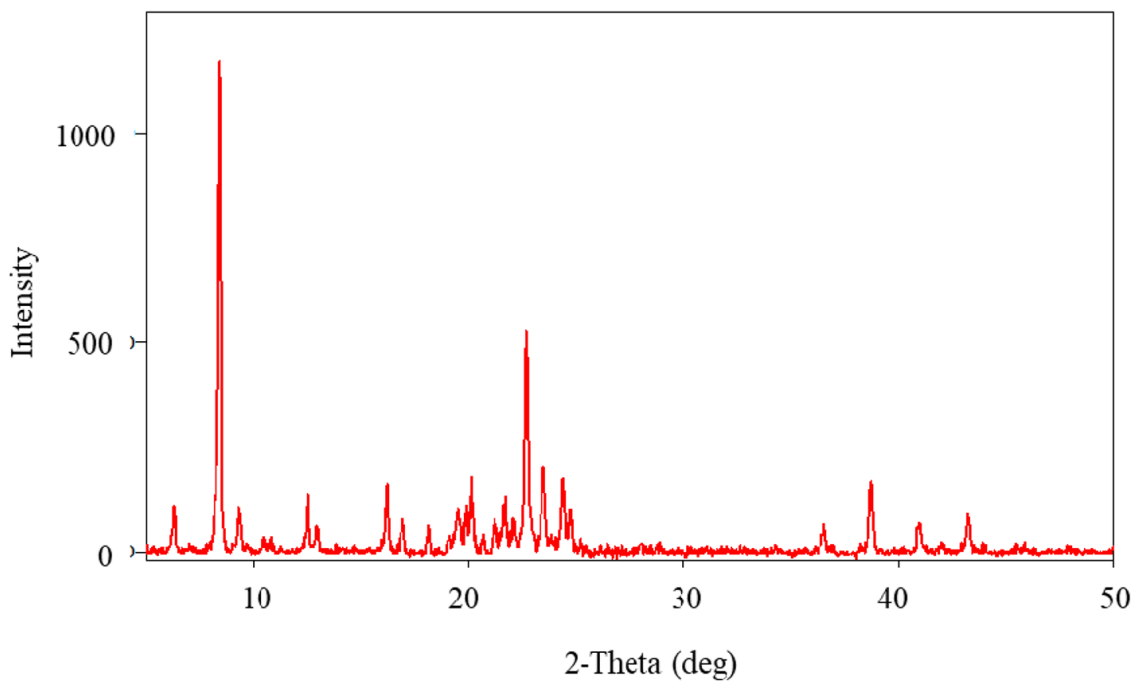

Fig. 6. XRD pattern of methyl 6-O-(3-bromobenzoyl)-2,3,4-tri-O-lauroyl- $\alpha$-D-mannopyranoside (8) 
The XRD patterns of the pure synthesized compounds under optimized conditions were displayed in the $2 \theta$ range of $\left(0^{\circ}-50^{\circ}\right)$. The peaks at $2 \theta$ value corresponding to $5.391 \& 21.846$ (h,k,l: $110 \& 400) ; 8.464 \& 22.654$ (h,k,l: $110 \& 220)$ and $9.799 \& 19.617$ (h,k,l: 110 \& 122) for compounds 7, 8 and 10 respectively. These peaks indicated the formation of typical phases of compounds 7, 8 and $\mathbf{1 0}$. According to the phase analysis, compounds synthesized under this method have high purity and no impurities were detected in the XRD pattern. In addition by

Table 3. Peak lists of the compounds $\mathbf{7 , 8}$ and $\mathbf{1 0}$

\begin{tabular}{|c|c|c|c|c|c|c|c|c|}
\hline $\begin{array}{c}\text { Compound } \\
\text { No }\end{array}$ & $\begin{array}{l}\text { Relative } \\
\text { intensity }\end{array}$ & $\begin{array}{c}2 \theta \\
(\operatorname{deg})\end{array}$ & $\begin{array}{c}\theta \\
(\operatorname{deg} .)\end{array}$ & $\operatorname{Sin}^{2} \theta$ & Ratio & $\mathrm{h}^{2}+\mathrm{k}^{2}+\mathrm{l}^{2}$ & (h k l) & d (ang) \\
\hline \multirow{5}{*}{7} & V. Strong & 5.391 & 2.6955 & 0.0022 & 1 & 100 & 100 & 16.38 \\
\hline & Weak & 10.916 & 5.8805 & 0.0091 & 4 & 112 & 112 & 8.099 \\
\hline & Strong & 21.846 & 10.923 & 0.0359 & 16 & 400 & 400 & 4.065 \\
\hline & Strong & 22.351 & 11.1755 & 0.3375 & 17 & 223 & 223 & 3.974 \\
\hline & Medium & 23.099 & 11.5495 & 0.040 & 18 & 330 & 330 & 3.847 \\
\hline \multirow{5}{*}{8} & V. Strong & 8.464 & 4.232 & 0.0054 & 1 & 100 & 100 & 10.438 \\
\hline & Strong & 22.654 & 11.327 & 0.0385 & 8 & 220 & 220 & 3.9218 \\
\hline & Weak & 23.449 & 11.7245 & 0.0412 & 8 & 220 & 220 & 3.791 \\
\hline & Weak & 24.383 & 12.1915 & 0.0445 & 8 & 220 & 220 & 3.647 \\
\hline & Weak & 38.702 & 19.351 & 0.1097 & 20 & 224 & 224 & 2.324 \\
\hline \multirow{4}{*}{10} & Weak & 9.799 & 4.8995 & 0.0073 & 1 & 100 & 100 & 9.019 \\
\hline & V. Strong & 19.617 & 9.8085 & 0.0290 & 4 & 122 & 122 & 4.521 \\
\hline & Weak & 20.267 & 10.1317 & 0.0309 & 4 & 122 & 122 & 4.3780 \\
\hline & Weak & 20.794 & 10.397 & 0.0325 & 4 & 122 & 122 & 4.268 \\
\hline
\end{tabular}

applying the rules (Table 1) for the determination of the lattice type, we have assigned the lattice structure of the synthesized compounds. It was found that compound $\mathbf{8}$ was satisfied the rule, $\mathrm{h}+\mathrm{k}+\mathrm{l}=2 \mathrm{n}$ and determined as a body-centered lattice and compound 7 also satisfied the rule, $\mathrm{h}+\mathrm{k}=2 \mathrm{n}$ and ascertained as the side-centered lattice. Besides the compound $\mathbf{1 0}$ wasdiscerned as a primitive typein which no rule was followed.

\section{Conclusion}

In this paper, regioselective3-bromobenzoylation of methyl $\alpha$-D-mannopyranoside (1) by applying the efficient direct method was unique in that the reaction provided a single mono-substitution product in reasonably good yields. The 3-bromobenzoylderivative 2 was further derivatized using a series of acyl chlorides. These acyl chlorides were deliberately chosen to introduce probably biologically prone atoms or groups to find biologically active D-mannopyranoside derivatives. Thus, structural modifications of the most active acylated derivatives determined in this study might provide favorable target compounds for further studies as potential antimicrobial agents. 


\section{Author contributions}

S.M.A.K. designedthe whole study; F.Y and M.R.A. performed the synthetic experiment and A.H. accomplished XRD analysis. S.M.A.K. interpreted the spectral data and wrote the manuscript. All authors have read and approved the final version of this paper.

\section{Acknowledgments}

The authors are indebted to the Ministry of Science and Technology (MOST) Government of Bangladesh, for financial assistance to carry out this research work [Ref: 39.00.0000.009.06.009.201331/Phy's-530, dated: 8-12-2020]. The authors are grateful to the Director, Wazed Miah Science Research Centre, JU, and Dhaka, Bangladesh, for recording the spectra.

\section{Declaration of interest}

The authors declare no conflict of interest.

\section{References}

1. Nicolaou K.C., Mitchell H.J. Adventures in carbohydrate chemistry: new synthetic technologies, chemical synthesis, molecular design, and chemical biology. Angewandte Chemie 2001. Vol. 40(9), P. 1576-1624.

2. Weymouth-Wilson A.C. The role of carbohydrates in biologically active natural products. Natural Product Report 1997. Vol. 14(2), P. 99-110.

3. Bertozzi C.R., Kiessling L.L. Chemical glycobiology. Science 2001. Vol. 291(5512), P. 2357-2364.

4. Codée J.D.C., Asghar A., Overkleeft H.S., van der Marel G.A. Novel protecting groups in carbohydrate chemistry. Comptes Rendus Chimie 2011. Vol. 14(2-3), P. 178-193.

5. Gornik O., Dumić J., Flögel M., Lauc G. Glycoscience- a new frontier in rational drug design. Acta Pharmaceutica 2006. Vol. 56, P. 19-30.

6. Williams J.M., Richardson A.C. Selective acylation of pyranosides-I. Benzoylation of methyl $\alpha$-D-glycopyranosides of mannose, glucose and galactose. Tetrahedron 1967. Vol. 23, P. 1369-1378.

7. Kim S., Chang H., Kim W. Regioselective acylation of some glycopyranoside derivatives. Journal of Organic Chemistry 1985. Vol. 50, P. 1751-1752.

8. Kawsar S M.A., Uddin S., Manchur M.A., Fujii Y., Ozeki Y. Acylation of D-glucose derivatives over $\mathrm{C}_{5} \mathrm{H}_{5} \mathrm{~N}$ : spectral characterization and in vitroantibacterial activities. International Journal of Biological Chemistry 2015. Vol. 9, P. 269-282.

9. Kawsar S.M.A., Faruk M.O., Rahman M.S., Fujii Y., Ozeki Y. Regioselective synthesis, characterization and antimicrobial activities of some new monosaccharide derivatives. Scientia Pharmaceutica 2014. Vol. 82(1), P. 01-20.

10. Ichinari M., Nakayama K., Hayase Y. Synthesis of 2,4-dioxoimidazolidines from 2-arylimino1,3-thiazines and their antifungal activity. Heterocycles 1988. Vol. 27, P. 2635-2641.

11. Gawande N.G., Shingare M.S. Synthesis of some thiazolylthiosemicarbazides, triazoles, oxazoles, thiadiazoles \& their microbial activity. Indian Journal of Chemistry 1987. Vol. 26, P. 387-394.

12. Gupta R., Paul S., Gupta A.K., Kachroo P.L., Bani S. Synthesis and biological activities of some 2-substituted phenyl-3-(3-alkyl/aryl-5,6-dihydro-s-triazolo[3,4-b][1,3,4]thiazolo-6-yl)-indoles. Indian Journal of Chemistry 1997. Vol. 36, P. 707-710. 
13. Singh H., Shukla K.N., Dwivedi R., Yadav L.D.S. Cycloaddition of 4-amino-3-mercapto1,2,4-triazole to heterocumulenes and antifungal activity of the resulting 1,2,4-triazolo[3,4-c]-1,2dithia-4,5-diazines. Journal of Agricultural \& Food Chemistry 1990. Vol. 38, P. 1483-1486.

14. Misbah M.M.H., Ferdous J., Bulbul M.Z.H., Chowdhury T.S., Dey S., Hasan I., Kawsar S.M.A. Evaluation of MIC, MBC, MFC and anticancer activities of acylated methyl $\beta$-D-galactopyranoside esters. International Journal of Biosciences 2020. Vol. 16(4), P. 299-309.

15. Islam M.M., Arifuzzaman M., Rahman M.M., Rahman M.A., Kawsar S.M.A. Novel methyl 4,6-O-benzylidene-a-D-glucopyranosidederivatives: synthesis, structural characterization and evaluation of antibacterial activities. Hacettepe Journal of Biology and Chemistry 2019. Vol. 47(2), P. $153-164$.

16. Devi S.R., Jesmin S., Rahman M., Manchur M.A., Fujii Y., Kanaly R.A., Ozeki Y., Kawsar S.M.A. Microbial efficacy and two step synthesis of uridine derivatives with spectral characterization. ACTA Pharmaceutica Sciencia 2019. Vol. 57(1), P. 47-68.

17. Alam A., Hosen M.A., Hosen A., Fujii Y., Ozeki Y., Kawsar S.M.A. Synthesis, characterization, and molecular docking against a receptor protein Fim H of Escherichia coli (4XO8) of thymidine derivatives. Journal of the Mexican Chemical Society 2021. Vol. 65(2), P. 256-276.

18. Bulbul M.Z.H., Chowdhury T.S., Misbah M.M.H., Ferdous J., Dey S., Hasan I., Fujii Y., Ozeki Y., Kawsar S.M.A. Synthesis of new series of pyrimidine nucleoside derivatives bearing the acyl moieties as potential antimicrobial agent, Pharmacia 2021. Vol. 68(1), P. 23-34.

19. Rana K.M., Ferdous J., Hosen A., Kawsar S.M.A. Ribose moieties acylation and characterization of some cytidine analogs. Journal of Siberian Federal University Chemistry 2020. Vol. 13(4), P. 465-478.

20. Misbah M.M.H., Ferdous J., Bulbul M.Z.H., Chowdhury T.S., Kawsar S.M.A. An efficient and easy route for the synthesis of methylß-D-galactopyranoside derivatives. Journal of the Bangladesh Chemical Society 2019. Vol. 31(2), P. 49-57.

21. Maowa J., Alam A., Rana K.M., Dey S., Hosen A., Fujii Y., Hasan I., Ozeki Y., Kawsar S.M.A. Synthesis, characterization, synergistic antimicrobial properties and molecular docking of sugar modified uridine derivatives. Ovidius University Annals of Chemistry 2021. Vol. 32(1), P. 6-21.

22. Rahman M., Sanjida J., Devi S.R., Rahman M., Islam M., Hossain M.K., Kanaly A.R., Fuji Y., Hayashi N., Ozeki Y., Kawsar S.M.A. Selective acylation of some carbohydrate derivatives using the direct method. Journal of the Bangladesh Chemical Society 2017. Vol. 29(2), P. 21-28.

23. Kawsar S.M.A., Khaleda M., Refat A., Manchur M.A., Koide Y., Ozeki Y. Infrared, ${ }^{1} \mathrm{H}-\mathrm{NMR}$ spectral studies of some methyl 6-O-myristoyl- $\alpha$-D-glucopyranoside derivatives: assessment of antimicrobial effects. International Letters of Chemistry Physics and Astronomy 2015. Vol. 58, P. 122-136.

24. Said S.A.J., Anwar U.H., Abdul R.I., Mohammed, S.S. Use of X-ray powder diffraction for quantitative analysis of carbonate rock reservoir samples. Powder Technology 2007. Vol. 175, P. $115-121$.

25. Kawsar S.M.A., Ferdous J., Mostafa G., Manchur M.A. A Synthetic approach of D-glucose derivatives: spectral characterization and antimicrobial studies. Chemistry and Chemical Technology Journal 2014. Vol. 8(1), P. 19-27. 